\title{
An intensive study on the optical, rheological, and electrokinetic properties of polyvinyl alcohol-capped nanogold
}

\author{
Manoranjan Behera ${ }^{1}$
}

Received: 20 February 2015/ Accepted: 7 May 2015/Published online: 20 May 2015

(c) The Author(s) 2015. This article is published with open access at Springerlink.com

\begin{abstract}
Low-temperature-assisted wet chemical synthesis of nanogold (NG) using gold hydroxide, a new precursor salt in the presence of a macroscopic ligand poly(vinyl alcohol) PVA in water in the form of nanofluid, is reported for the first time in this article. In the absorption spectra, the surface Plasmon resonance absorption band in the range of 520-545 nm signifies the formation of NG via a controlled $\mathrm{Au}^{3+}+3 \mathrm{e} \rightarrow \mathrm{Au}$ reaction grafted in small assemblies with polymer. Absorption maximum increases nonlinearly with Au-contents up to $100 \mu \mathrm{M}$ Au in Au-PVA charge-transfer complex. Marked enhancement in the peak intensity of some of the vibration bands of PVA polymer such as $\mathrm{C}-\mathrm{H}$ stretching, $\mathrm{C}=\mathrm{O}$ stretching, $\mathrm{CH}_{2}$ bending, and $\mathrm{C}-\mathrm{C}$ in-plane bending in the presence of $\mathrm{NG}$ reveals an interfacial interaction between $\mathrm{NG}$ and oxidized PVA via $\mathrm{C}=\mathrm{O}$ group. Execution of shear thinning behavior regardless of the Au-content strongly suggests that crosslinking exists between NG and PVA in Au-PVA rheo-optical nanofluids. Hydrodynamic diameter and polydispersity index draw a nonlinear path with the Au doping with $30.0 \mathrm{~g} /$ L PVA in water over a wide region of $5-100 \mu \mathrm{M} \mathrm{Au}$ covered in this study. Enhancement in the zetapotential of Au-PVA nanofluid over bare PVA in water is ascribed to buildup of nonbonding electrons of " $-\mathrm{C}=\mathrm{O}$ " moieties from the oxidized PVA on the NG surface. Displaying of lattice fringes in the microscopic image of core-shell Au-PVA nanostructure confirms that crystalline nature of NG core with inter planar spacing $0.235 \mathrm{~nm}$ corresponds to $\mathrm{Au}$ (111) plane.
\end{abstract}

Manoranjan Behera

mano.silicon@gmail.com

1 Faculty in Chemistry, Silicon Institute of Technology, Silicon Hills, C S PUR, Bhubaneswar 751 024, Odisha, India
Keywords Nanofluids - Dynamic light scattering · Electrokinetic effect · Interfacial interaction · Core-shell nanostructure

\section{Introduction}

Nanofluids (NFs) represent a relatively new class of functional and engineered materials which consist of nanoscale materials (2-50 nm size) such as metals $(\mathrm{Cu}, \mathrm{Ag}, \mathrm{Au}, \mathrm{Pt}$, etc.), oxides $\left(\mathrm{Al}_{2} \mathrm{O}_{3}, \mathrm{SiO}_{2}, \mathrm{Fe}_{3} \mathrm{O}_{4}, \mathrm{TiO}_{2}\right.$, etc.), carbides ( $\mathrm{SiC}$, $\mathrm{WC}$, etc.), carbon (fullerenes, graphene, and carbon nanotubes) dispersed in water, mineral oils, ethylene glycol, polymer solutions, and biofluids imperative for diverse applications of medicines, color pigments, biological sensors, energy transfer, coolants, and many others [1-7]. The innovative idea of the NFs was first introduced by Dr. Choi of Argonne National Laboratory as early as 1995 [1].

Soon after the synthesis of nanogold (NG) by Michael Faraday in 1857 [8], NFs containing noble metal nanoparticles (NPs) have been attracting extensive attention in scientific communities on account of potential applications of NG in the various fields such as sensing [9-12], biomedicals [9-13], catalysis [9-12], photonics [11-13], memory devices [11-13], and heat transfer [14, 15]. The synthesis of metal-polymer NFs with controlled microstructure and functional properties is an integral part of an emerging and rapidly growing "materials technology". The efforts are being made on possibility to control over the microstructures via innovative synthetic methods. In this regard, various bottom-up approaches, such as photolysis [10, 13], chemical reduction [12, 16], and template methods [17, 18], have been adopted for the synthesis of NG of controlled shapes and sizes. Moreover, in an in situ technique, a nanostructured metal is produced within a polymer either 
via a chemical decomposition of a metal complex or by a chemical reduction reaction of metal salts by using a proper reducing agent in a solvent. In particular, NG of various architectures such as rods, plates, disks, cubes, spheres, stars, etc. can be synthesized by proper manipulation of experimental parameters in the presence of a macroscopic shape-regulating agents in the form of polymer [9-13, 16].

Commonly used polymers for surface stabilizing noble metals like gold in a nanostructure in a liquid medium include poly(vinyl pyrrolidone) PVP [13, 19-24], poly(ethylene glycol) PEG [15], poly(vinyl alcohol) (PVA) [9, 11, $12,16]$, polymethyl methacrylate [10], and poly(vinylidene fluoride) [25, 26]. Colloidal stability in NFs can be achieved by surrounding particles (1) with an electrical double-layer (i.e., an electrostatic stabilization), (2) by adsorbing or chemically attaching polymeric molecules (i.e., a steric stabilization), or (3) by a free polymer in the dispersion medium (i.e., depletion stabilization) [5, 6, 27, 28]. Combination of the first two stabilization mechanisms leads to electrosteric stabilization. So far as the electrostatic stabilization is concern, particles with zetapotential $(\xi) \geq( \pm) 30$ $\mathrm{mV}$ are normally considered as stable nanofluid [27, 28].

Herein, we discuss a simple in situ synthesis method to develop NG from gold hydroxide, a relatively new precursor salt [20-24], in the presence of a macroscopic stabilizer and reducer like polyvinyl alcohol (PVA) in an aqueous medium. To the best of our knowledge, this is the first report on synthesis of NG using gold hydroxide as precursor salt in an aqueous medium at relatively low reaction temperature of $40{ }^{\circ} \mathrm{C}$. Porel et al. [9] have synthesized polygonal $\mathrm{Au}$ nanoplates using $\mathrm{HAuCl}_{4}$ salt in the presence of PVA by a thermal treatment at $100-170{ }^{\circ} \mathrm{C}$. In an another experiment, Tripathy et al. [16] reported synthesis of nanoplates of $\mathrm{Au}$ of various architectures using $\mathrm{HAuCl}_{4}$ salt in the presence of PVA via an in situ wet chemical route at $50-60{ }^{\circ} \mathrm{C}$. As our salt gives free $\mathrm{Au}^{3+}$ ions in solution whose reduction potential is less (i.e., $\left.\mathrm{Au}^{3+}+3 \mathrm{e} \rightarrow \mathrm{Au}, E^{0}=0.8 \mathrm{~V}\right)$ as compared to widely used $\mathrm{HAuCl}_{4}$ salt (i.e., $\mathrm{AuCl}_{4}{ }^{-}+3 \mathrm{e} \rightarrow \mathrm{Au}+4 \mathrm{Cl}^{-}$, $E^{0}=1.1 \mathrm{~V}$ ), reduction from $\mathrm{Au}^{3+}$ ions to $\mathrm{Au}$ atom is easier and hence reduction reaction occurs at a low temperature. The samples were characterized using UV-Visible, parallel plate rotational rheometer, zetapotential, dynamic light scattering (DLS), and high-resolution transmission electron microscope (HRTEM).

\section{Experimental details}

\section{Materials}

Reagent grade $\mathrm{Au}(\mathrm{OH})_{3}$ powder was purchased from Alfa Aesar and was dissolved in dilute $\mathrm{HNO}_{3}$ to prepare a stock solution of $2 \mathrm{mM} \mathrm{Au}\left(\mathrm{NO}_{3}\right)_{3}$. Polyvinyl alcohol (PVA) of average molecular weight $\sim 125,000$ was purchased from Sigma Aldrich. All the chemicals were used as received without further purification.

\section{Synthesis of PVA-Au NFs in water}

At first, an aqueous solution of PVA (30 g/L) was prepared in water by magnetic stirring for $3 \mathrm{~h}$ at $60-70{ }^{\circ} \mathrm{C}$. After this, a specific volume of gold nitrate solution was added drop wise while stirring to a $5 \mathrm{~mL}$ of $30 \mathrm{~g} / \mathrm{L}$ PVA solution in water at $40{ }^{\circ} \mathrm{C}$. Light purple-colored NFs were obtained after magnetic stirring the solution for $5 \mathrm{~min}$ and then sonicated at $40{ }^{\circ} \mathrm{C}$ in an ultrasonicator (OSCAR, $20 \mathrm{kHz}$ frequency and $250 \mathrm{~W}$ power) for $5 \mathrm{~min}$. PVA-Au NFs thus obtained were studied using experimental techniques such as UV-Visible, Fourier transform infrared (FTIR) spectrometer, rheometer, DLS, zeta potential, and HRTEM.

\section{Experimental techniques}

The optical absorption spectra of aqueous $30 \mathrm{~g} / \mathrm{L}$ PVA solution with and without NG were measured under identical conditions on a Perkin-Elmer double beam spectrophotometer (LAMBDA 1050). The sample was filled in a transparent cell of quartz (10-mm optical length) and the spectrum was recorded against a reference (water, or $30 \mathrm{~g} / \mathrm{L}$ PVA with water) in an identical cell. Vibration spectra of the solution have been studied with a Thermo Nicolet Corporation FTIR spectrometer (Model NEXUS-870). The spectra have been recorded in an attenuated total reflectance mode using a $\mathrm{ZnSe}$ crystal as a sample holder. Zetapotential $(\xi)$, hydrodynamic diameter $\left(L_{\mathrm{hd}}\right)$, and polydispersity index (PDI) were measured by using a Malvern Nano ZS instrument using phase analysis light scattering technique. Diluted samples were sonicated for $60 \mathrm{~s}$ prior to measurements. The samples were analyzed three times at $25{ }^{\circ} \mathrm{C}$. The rheological properties of Au-PVA NFs of varied compositions in water were measured using a rotational rheometer (TA instruments, model: AR-1000) of parallel plate geometry, with an upper plate of diameter $40 \mathrm{~mm}$. To measure the data, a few drops of the fluid were put on the lower plate of the system with a torque varying from 0.2 to $0.5 \mu \mathrm{N}-\mathrm{m}$ at shear rates $\gamma$ varying from 10 to $500 \mathrm{~s}^{-1}$. The shear viscosity and shear stress were measured at selective $\gamma$ values in the 10-500 $\mathrm{s}^{-1}$ range. Microscopic image of PVAcapped Au was studied with a JEM-2100 (JEOL, Japan) instrument. HRTEM samples were prepared by placing one drop of diluted solution on a carbon coated 400-mesh copper grid and allowing the sample to dry in desiccators at room temperature. 


\section{Results and discussion}

\section{UV-Visible and FTIR spectra}

The UV-Visible spectra were studied in the Au-PVA NFs in the 200-900 nm regions. We varied Au-content from 5 to $100 \mu \mathrm{M}$ under identical conditions of the experimental parameters in order to compare and analyze the results upon incorporating NG. As shown in the inset of Fig. 1, the absorption spectrum of PVA polymer $(3.0 \mathrm{~g} / \mathrm{dL}$ in water) solution absorb strongly in three distinct bands of 231, 281 and $324 \mathrm{~nm}$ wavelength of the absorption maximum $\left(\lambda_{\max }\right)$. The three bands belong to the $\pi \leftarrow \pi^{*}$ electronic transitions in PVA in three different tacticities, namely syndiotactic-PVA, atactic-PVA, and isotactic-PVA, respectively [29]. No absorption from PVA in the visible region of the spectrum was observed without NG (Fig. 1a). In the spectrum in Fig. $1 \mathrm{~b}$, in a $5 \mu \mathrm{M}$ Au-PVA nanofluid, one can see a new band developed in the $450-750 \mathrm{~nm}$ regions, with $\lambda_{\max }$ at $522 \mathrm{~nm}$ in the presence of NG. This strong absorption band occurs due to the collective oscillation of the conduction $5 \mathrm{~d}^{10} 6 \mathrm{~s}^{1}(\mathrm{Au})$ electrons in dispersed NG via the PVA polymer in response to the optical excitation, i.e., the surface Plasmon resonance (SPR) band $[11,12,16,19-24,30,31]$. The SPR absorption band signifies the formation of $\mathrm{NG}$ via a controlled $\mathrm{Au}^{3+}+3-$ $\mathrm{e} \rightarrow \mathrm{Au}$ reaction embedded in small assemblies of polymer. It is also seen that reinforcing PVA polymer using NG very sensitively tunes the absorption profile in the Au-SPR band over the $500-750 \mathrm{~nm}$ spectral region. In the Scheme 1, I proposed a scheme to show how the $-\mathrm{OH}$ group of PVA polymer reduced $\mathrm{Au}^{3+}$ ions to $\mathrm{Au}$ atoms and itself converted into $-\mathrm{C}=\mathrm{O}$ group to convert into oxidized

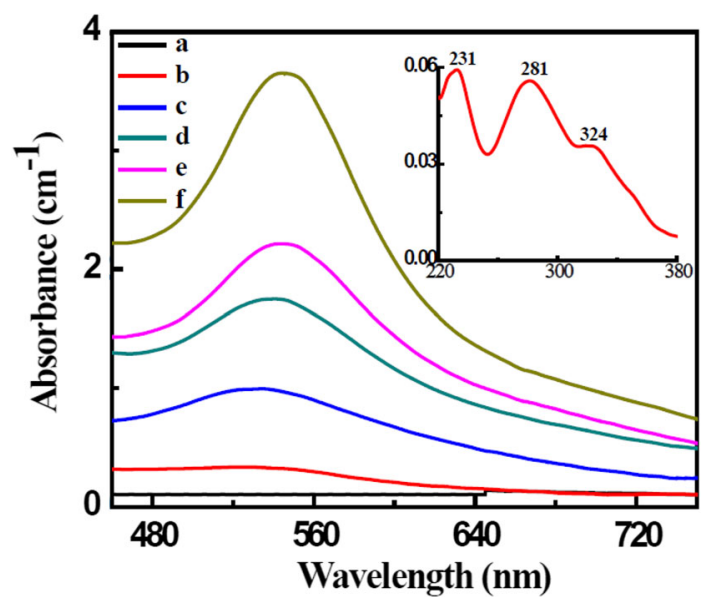

Fig. 1 Absorption spectra in Au-PVA NFs consisting of $a 0, b 5$, $c 10, d 20, e$ 50, and $f 100 \mu \mathrm{M}$ Au with $30.0 \mathrm{~g} / \mathrm{L}$ PVA in water. Inset shows an absorption spectrum of $30.0 \mathrm{~g} / \mathrm{L}$ PVA in water in the UV region

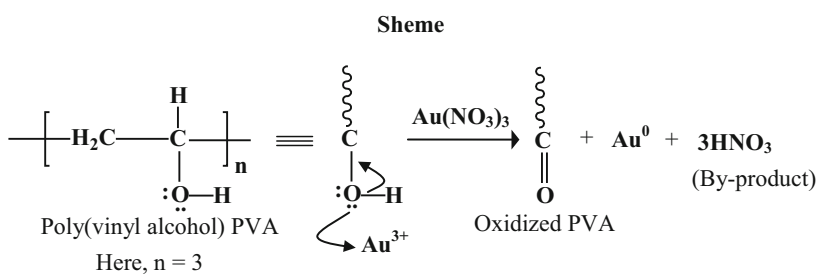

Scheme 1 Formation of nanogold from gold nitrate in presence of PVA

PVA polymer. The $\lambda_{\max }$ value for SPR band undergoes a red-shift to a value $533 \mathrm{~nm}$ upon raising the $\mathrm{Au}$-content to $10 \mu \mathrm{M}$ while to $544.2 \mathrm{~nm}$ when Au-content has increased to $100 \mu \mathrm{M}$. In such organic-inorganic nanocomposite, redshift in $\lambda_{\max }$ value for SPR band with Au-content may be ascribed to the various reasons such as the effective particle shape, the surface topology (especially of a core-shell structure), average inter-particle distance, average dielectric constant, and the microstructure of the medium surrounding the metal NPs [31].

The variations of the absorption maximum $\left(\alpha_{\max }\right)$ as a function of the Au-content in the Au-PVA NFs are portrayed in Fig. 2. The $\alpha_{\max }$ value follows a nonlinear path over Au-contents owing to charge transfer from PVA to NG. In the plot made in this figure, the $\alpha_{\max }$ value passes through a critical value $25.0 \mu \mathrm{M}$ Au-content near point B and then starts increasing with a further increase in the $\mathrm{Au}-$ content over the subsequent region covered in this experiment. The point $\mathrm{B}$ indicates a percolation effect of the microscopic bridging and interaction between the two components NG and PVA in a nanofluid.

The FTIR spectra measured in the transmittance mode were used in aiming to comment on the effects of the NG on the local structures of PVA in the NF. As expected, only the selective IR bands in the normal modes of the vibration of PVA show a noticeable change in the frequency or peak intensity upon addition of NG. The spectra consist of wellresolved bands over $500-1800 \mathrm{~cm}^{-1}$ followed by a broad band over $2000-4000 \mathrm{~cm}^{-1}$. Figure 3 shows the FTIR spectra $3.0 \mathrm{~g} / \mathrm{dL}$ PVA (a) before and (b) after doping of a typical $5 \mu \mathrm{M} \mathrm{Au}$ in water. Marked enhancement in the peak intensity of some of the vibration bands of PVA polymer such as $\mathrm{C}-\mathrm{H}$ stretching $\left(2975\right.$ and $\left.2861 \mathrm{~cm}^{-1}\right)$, $\mathrm{C}=\mathrm{O}$ stretching $\left(1725 \mathrm{~cm}^{-1}\right), \mathrm{CH}_{2}$ bending $\left(793 \mathrm{~cm}^{-1}\right)$, and $\mathrm{C}-\mathrm{C}$ in-plane bending $\left(1008\right.$ and $\left.1070 \mathrm{~cm}^{-1}\right)$ in the presence of $5 \mu \mathrm{M}$ NG reveals interfacial interaction between NG and PVA via $\mathrm{C}=\mathrm{O}$ group. Similar observations had already reported in Au and poly(vinyl pyrrolidone) PVP polymer in aqueous and non-aqueous media [20-24]. The presence of a residual acetate group often reflects in $\mathrm{C}=\mathrm{O}$ stretching band in such polymer [32]. A substantial enhancement in the peak intensity and band broadening of 


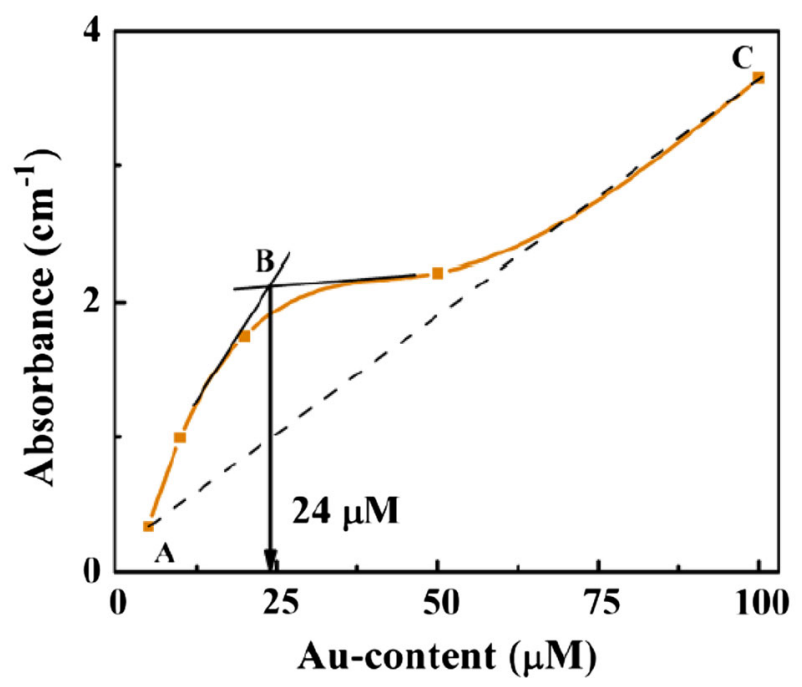

Fig. 2 Variation of absorbance with Au-content in Au-SPR band in Au-PVA NFs with $30.0 \mathrm{~g} / \mathrm{L}$ PVA in water. Absorbance increasing with Au-content up to point ' $C$ ' before passing through a shoulder

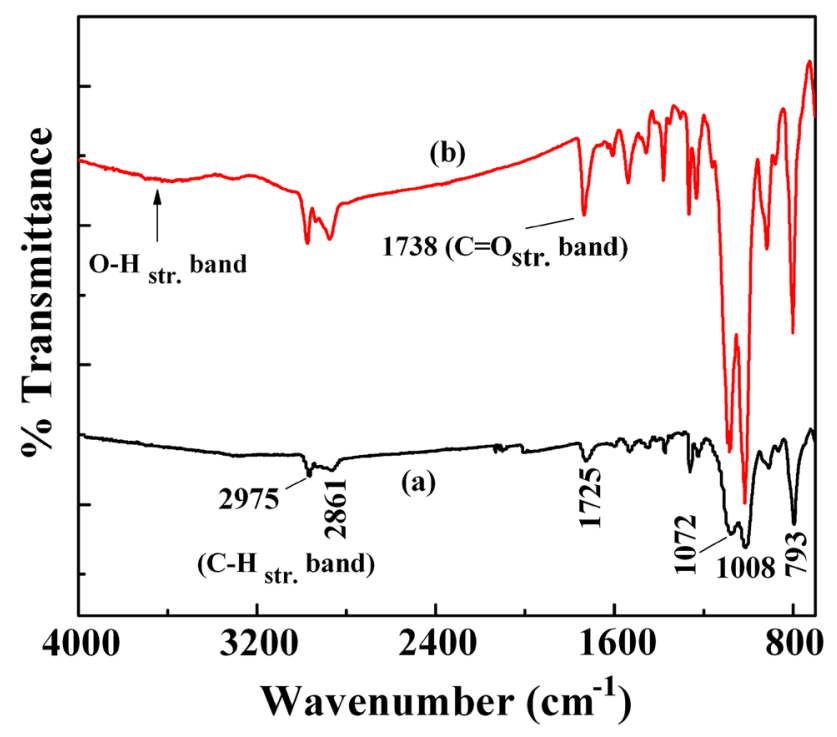

Fig. 3 FTIR spectra of $a 30.0 \mathrm{~g} / \mathrm{L}$ PVA in water and $b$ Au-PVA nanofluid consisting of $5 \mu \mathrm{M} \mathrm{NG}$ with $30.0 \mathrm{~g} / \mathrm{L}$ PVA in water

$\mathrm{C}=\mathrm{O}$ stretching band in the presence of $\mathrm{NG}$ evidence conversion of some $\mathrm{C}-\mathrm{OH}$ group in the PVA matrix into $\mathrm{C}=\mathrm{O}$ during the process of reducing $\mathrm{Au}^{3+}$ to $\mathrm{Au}$ atoms with reactive PVA which play active roles of a reducing agent in forming NG [33, 34]. The Scheme 1 as discussed in the earlier section shows that $-\mathrm{OH}$ group of PVA polymer has oxidized to $-\mathrm{C}=\mathrm{O}$ group in the process of formation of $\mathrm{Au}$ atoms. Further, a blue-shift in the $\mathrm{C}=\mathrm{O}$ stretching band from a value $1725 \mathrm{~cm}^{-1}$ in the PVA to a value $1738 \mathrm{~cm}^{-1}$ in the Au-PVA nanofluid reveals an interfacial interaction between $\mathrm{Au}$ and $\mathrm{C}=\mathrm{O}$ group of the oxidized PVA polymer. In an experiment, Grace and Pandian [35] have reported a small blue-shift (about $10 \mathrm{~cm}^{-1}$ ) in the $\mathrm{C}=\mathrm{O}$ stretching frequency of PVP polymer in the presence of NG owing to a surface interaction between the $\mathrm{C}=\mathrm{O}$ group of $\mathrm{PVP}$ and $\mathrm{Au}-\mathrm{NPs}$.

\section{Rheology in Au-PVA NFs}

Now let us comment on how average shear viscosity $(\eta)$ of an Au-PVA nanofluid correlates to its optical properties of the light absorption in the PVA and Au if they are grafted in joint assemblies. Figure 4 plots the results of $\eta$ values measured as a function of shear rate $(\gamma)$ in the various AuPVA NFs consisting of (a) 5, (b) 10, (c) 20, (d) 50, and (e) $100 \mu \mathrm{M}$ Au. The initial $\eta$ values in all the samples decay rapidly over early shear rates until $150 \mathrm{~s}^{-1}$ before the ultimate entities (flocculates) become rather more resistive in a way that they lose the final $\eta$ values steadily over subsequently higher shear rates over $150-500 \mathrm{~s}^{-1}$. All the samples describe a shear thinning behavior regardless of the Au-content and reveal that Au NPs are crosslinking to PVA to form joint assemblies. Mishra et al. [36] proposed a similar grafted stricture of Au:PVP dispersed in water. Recently, Phule et al. [37] reported that a rigid structure builds up in nanosilver cross-links to poly (vinylidene fluoride) and it results in an enhanced $\eta$ value over the base value in a bare polymer solution. As shown in the inset of Fig. 4, as expected, water is exhibiting Newtonian behavior, whereas $30.0 \mathrm{~g} / \mathrm{L}$ PVA in water is showing a non-Newtonian behavior due to interaction between PVA and water molecules.

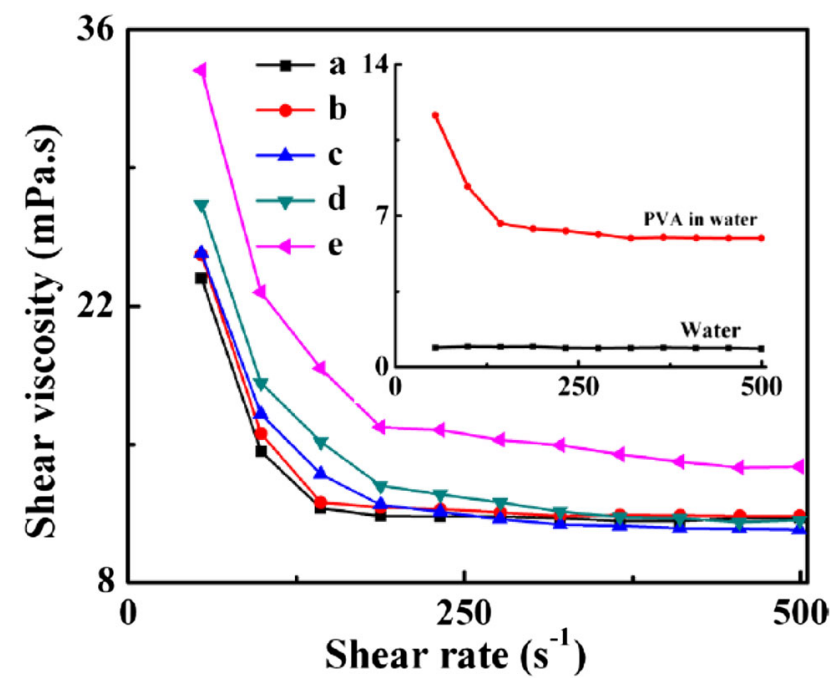

Fig. 4 The variation of shear viscosity as a function of shear rate in Au-PVA NFs with $30.0 \mathrm{~g} / \mathrm{L}$ PVA in water; $a 5, b 10, c 20, d$ 50, and e $100 \mu \mathrm{M} \mathrm{Au}$. Inset shows the rheogram for water and $30.0 \mathrm{~g} / \mathrm{L}$ PVA in water 
A variation of shear stress $(\tau)$ against the $\gamma$ value in the various Au-PVA NFs in water is portrayed in Fig. 5. A linear plot shown in all the samples with a residual yield stress $\left(\tau_{y}\right)$ is a signature of the Bingham flow [37]. The experimental points in Fig. 5 fit very well with the characteristic equation of a Bingham plastic [37]. From linear fit, we have found that a $\tau_{\mathrm{y}}$ value has increased from $387 \mathrm{mPa}$ in Sample-a to $1047 \mathrm{mPa}$ in Sample-e which contains as large as $100 \mu \mathrm{M} \mathrm{NG}$ in water. This result suggests that $\mathrm{Au}$ NPs are extensively crosslinking to PVA in such a way that the assemblies give a nonzero $\tau_{\mathrm{y}}$ value. In a review on rheology, Muller et al. [38] reported that a $\tau_{\mathrm{y}}$ value develops in suspensions of solid particles when they form chains and network structures. Variation of $\gamma$ values with Au-content in the Au-PVA NFs with a fixed $30.0 \mathrm{~g} / \mathrm{L}$ PVA in water at a selective shear rate $200 \mathrm{~s}^{-1}$ is shown as inset in Fig. 5. The $\eta$ values, which decrease with $\gamma$ value over 5-500 s $\mathrm{s}^{-1}$, are shown to be increasing nonlinearly against the Au-content, tracing out a weak minima near $26 \mu \mathrm{M} \mathrm{NG}$ in the percolation effect, as it has been described above in the light absorption spectra in these samples. At this particular Au-content, a relative decrease in the viscosity possibly helps acquire a relatively enhanced absorbance value in this sample.

\section{DLS and Zetapotential in Au-PVA NFs}

The DLS also known as photon correlation spectroscopy is a sensitive technique which provides information on size in terms of hydrodynamic diameter $\left(L_{\mathrm{hd}}\right)$ and width of distribution (i.e., PDI) of $\mathrm{Au}$ and Au-grafted polymer hybrids in a nanofluid. Figure 6 depicts the normalized autocorrelation function $g^{1}(\tau)$ values measured for Au-PVA NFs comprising (a) 0, (b) 5, (c) 9.26, (d) 10, (e) 20, (f) 50, and

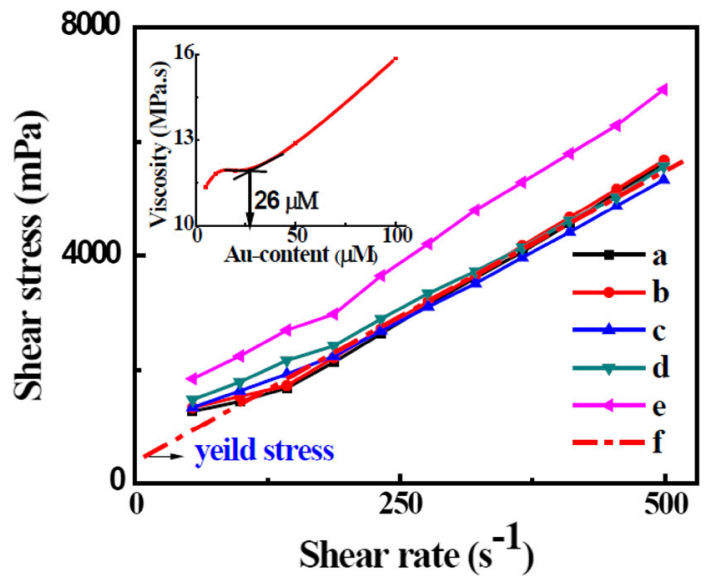

Fig. 5 Variation of shear stress as a function of shear rate in Au-PVA NFs; $a 5, b 10, c 20, d 50$, and $e 100 \mu \mathrm{M} \mathrm{Au}$. " $f$ " is the linear fit to sample $a$. Inset shows the variation of shear viscosity against Aucontent in Au-PVA NFs at $200 \mathrm{~s}^{-1}$ shear rate (f) $100 \mu \mathrm{M} \mathrm{NG}$ along with $30.0 \mathrm{~g} / \mathrm{L}$ PVA in water. As it can be seen from the plot in Fig. 6, with increase in the Aucontent, the correlogram shifts right along the $X$-axis. This reveals that an $\mathrm{Au}$ doping alters the $L_{\mathrm{hd}}$ value in a nanofluid and as a result has an effect on the delay time $\tau$. For example, in sample (f) which contains as large as $100 \mu \mathrm{M}$ with $30.0 \mathrm{~g} / \mathrm{L}$ PVA in water, owing to effectively large NG cluster size, the signal has changed slowly and the correlation had persevered for a long time compared to sample (b). In a report on Au:PVP NFs in water, Mishra et al. [36] have reported a similar shift of the correlograms over larger $L_{\mathrm{hd}}$ values on increasing the Au-content from 2.54 to $50.76 \mathrm{mM}$, supporting a fact that added $\mathrm{Au}$ combines to PVP in joint assemblies.

Further, we studied DLS bands in the Au-PVA NFs prepared with the different Au-contents (a) 0, (b) 5, (c) 10, (d) 20, (e) 50, and (f) $100 \mu \mathrm{M}$ in $30.0 \mathrm{~g} / \mathrm{L}$ PVA in water. The experimental data could be collected over five times diluted samples which had an average $\mathrm{pH}$ of 7.5. As it is can be seen from the DLS spectra portrayed in Fig. 7, excluding sample-a for PVA in water, all the five samples exhibit two distinct DLS bands, indicating two kinds of size distributions of the agglomerates of $\mathrm{Au}$ and PVA in water. In sample (a), only PVA dispersed in water exhibits a single DLS band with an average $L_{\mathrm{hd}}$ value $16 \mathrm{~nm}$ and a PDI 0.252. It is observed that when only a small dosage of $5 \mu \mathrm{M} \mathrm{NG}$ is added to this sample, two well-separated bands split-up in two effective $L_{\mathrm{hd}}$ values 25.0 and $15.1 \mathrm{~nm}$. An average $L_{\mathrm{hd}}$ value $21 \mathrm{~nm}$ is estimated from the total band profile, with PDI 0.372. In all the five NFs, the first Band-1 attributes to PVA dispersed in water and the Band-2 arises in PVA-capped NG. This clearly hints that upon addition of $100 \mu \mathrm{M}$ Au to PVA in water, the Au-PVA

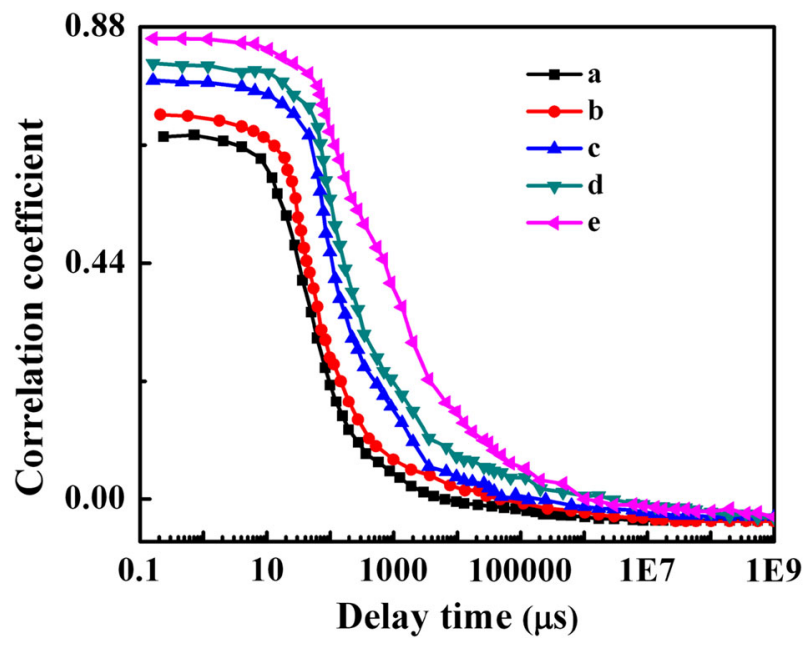

Fig. 6 Variations of normalize correlation coefficient values against the time scale of measurement in Au-PVA NFs in water; $a$ 5, $b 10$, $c 20, d 50$, and $e 100 \mu \mathrm{M} \mathrm{Au}$ 


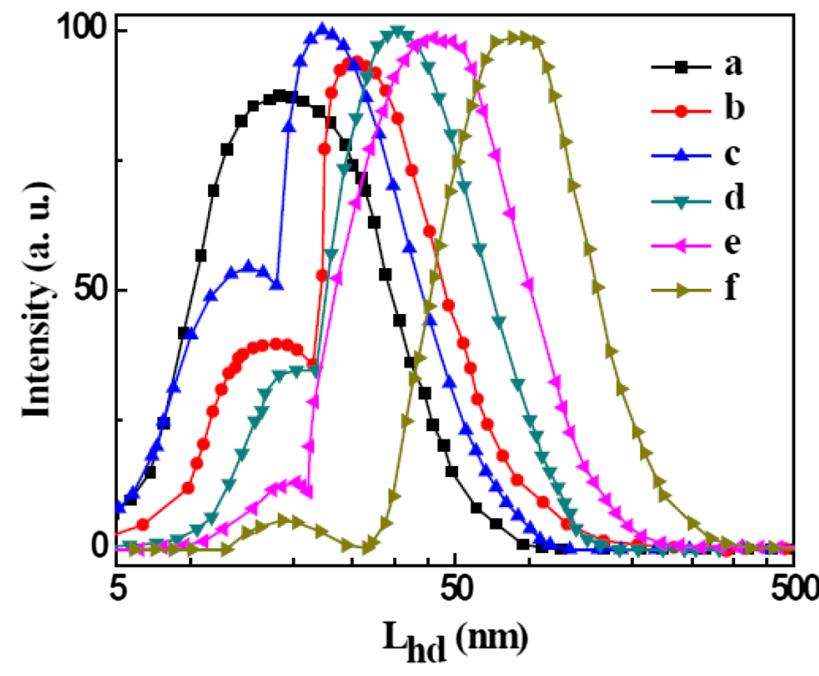

Fig. 7 Distributions of $L_{\mathrm{hd}}$ values in Au-PVA NFs consisting of $a 0$, $b 5, c 10, d 20, e 50$, and $f 100 \mu \mathrm{M} \mathrm{NG}$ in $30.0 \mathrm{~g} / \mathrm{L}$ PVA in water

Table 1 The values of $\xi, L_{\mathrm{hd}}$, and PDI determined from zeta potential and DLS for bare PVA solution and synthesized Au-PVA NFs in water

\begin{tabular}{llll}
\hline Au-content $(\mu \mathrm{M})$ & $\xi$ value $(\mathrm{mV})$ & $L_{\mathrm{hd}}$ value $(\mathrm{nm})$ & PDI value \\
\hline 0 & $(-) 8.0$ & 16 & 0.252 \\
5 & $(-) 23.2$ & 21 & 0.372 \\
10 & $(-) 22.0$ & 25 & 0.382 \\
20 & $(-) 25.0$ & 35 & 0.323 \\
50 & $(-) 21.0$ & 45 & 0.381 \\
100 & $(-) 16.2$ & 80 & 0.425 \\
\hline
\end{tabular}

The samples were diluted five times in water in measuring the data

cluster size has increased nonlinearly to as large as $80 \mathrm{~nm}$, with PDI 0.425 (Table 1). Mishra et al. [36] have reported only a linear growth in the $L_{\mathrm{hd}}$ value with Au-content in Au:PVP NFs prepared in water, possibly in difference in the process of forming the clusters.

Furthermore, we studied the variations in $L_{\mathrm{hd}}$ and PDI values with Au-content in the synthesized Au-PVA NFs in water. As the results are plotted in Fig. 8, both these physical parameters trace a nonlinear path with the $\mathrm{Au}$ doping of a $30.0 \mathrm{~g} / \mathrm{L} \mathrm{PVA}$ in a solution in water over a wide region of $5-100 \mu \mathrm{M} \mathrm{Au}$ covered in this study. It is evident from the plot in Fig. 8 that on increasing the Aucontent, the $L_{\mathrm{hd}}$ value displays a peak near $26.0 \mu \mathrm{M}$ NG in a percolation effect, as it was also shown in the light absorption study (discussed in Sect. 3.1). An increase in $L_{\mathrm{hd}}$ value with an increase in the Au-content is due to a decrease in the relative PVA content in the samples. In an experiment, Rahme et al. [39] reported that $L_{\mathrm{hd}}$ value of $\mathrm{Au}$ NPs has increased in a nonlinear way from 10 to $90 \mathrm{~nm}$ by increasing molecular weight of the polymer from 2100 to

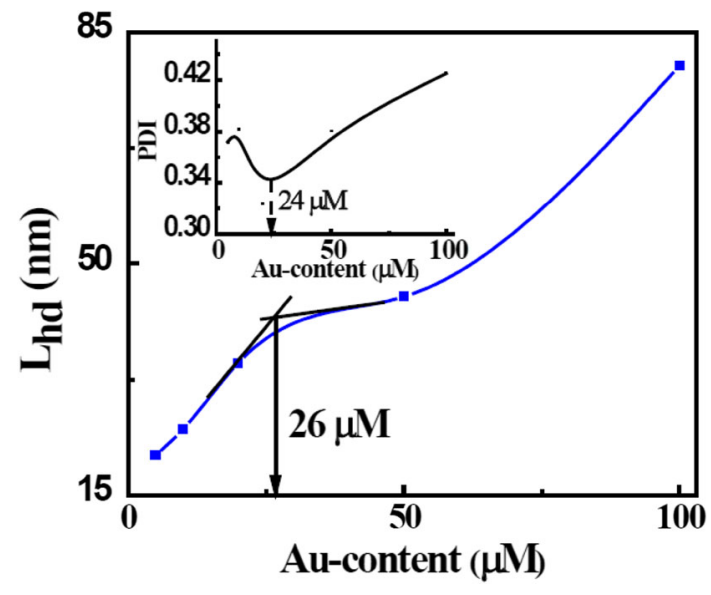

Fig. 8 Variations in average $L_{\text {hd }}$ value with Au-content in Au-PVA NFs prepared in $30.0 \mathrm{~g} / \mathrm{L}$ PVA in water. Inset shows variations in PDI value with $\mathrm{Au}-$ content

$51,400 \mathrm{~g} \mathrm{~mol}^{-1}$ in grafted Au-PEG NFs in water due to a decrease in grafting density from $3.93 \mathrm{~nm}^{2}$ to $0.32 \mathrm{~nm}^{2}$. Conversely, the PDI value Vs Au-content plot made in Fig. 8 as inset exhibits a dip near $24.0 \mu \mathrm{M}$ Au. As a result, a relatively small PDI value of 0.323 is obtained in sampled. It implies that both the Au-PVA and PVA entities are distributed reasonably uniformly in their effective sizes in the two distinct distribution bands in water in sample-d. It accounts well for observation of a reasonably lowered $\eta$ value and enhanced absorbance in the present Au-PVA NFs at this critical Au amount.

We studied distribution of $\xi$ values in Au-PVA NFs consisting of (a) 0, (b) 5, (c) 10, (d) 20, (e) 50, and (f) $100 \mu \mathrm{M} \mathrm{NG}$ along with $30.0 \mathrm{~g} / \mathrm{L}$ PVA in water. As the experimental data could be collected only from reasonably dilute samples, the samples were diluted five times (average $7.5 \mathrm{pH}$ ) before recording the data. As the spectra are shown in Fig. 9 for the various samples, a bare PVA solution in sample (a) exhibits a single sharp zeta band at an average $\xi$ value $(-) 8.0 \mathrm{mV}$ in water at $7.5 \mathrm{pH}$. In bare PVA polymer that is dispersed in water, a negative $\xi$ value results from the presence of nonbonding (n) electrons on the $-\mathrm{OH}$ sites. Moreover, addition of $5 \mu \mathrm{M}$ of $\mathrm{Au}$ NPs to sample (a) causes a broader zeta band of an average $\xi$ value increased to $(-) 23.2$. $\mathrm{mV}$. Such enhanced $\xi$ value is ascribed to amassing of n-electrons of " $-\mathrm{C}=\mathrm{O}$ " moieties from the oxidized PVA on the NG surface in Au-PVA assemblies. In our earlier experiments in $\mathrm{Au}$ and fullerene PVP NFs in water, we had reported that $\xi$ value of $\mathrm{Au}$ and fullerene had increased when n-electrons of $\mathrm{C}=\mathrm{O}$ moieties of PVP are accumulated on the surface of nanoparticles [20-24, 40].

We also studied how the $\xi$ value varies in these Au-PVA NFs with the Au-content in an aqueous medium. As depicted in the inset of Fig. 9, $\xi$ value shows peak maxima 
near $25 \mu \mathrm{M}$ Au which acquires a relatively small $\eta$ value with a reasonably decreased PDI index (Table 1) in the AuPVA NFs. As a result, effectively large $\xi$ value has a correlation to a reasonably low viscous sample. Such a sample lets a light-induced mechanical perturbation propagate through the sample. In the other words, a PVA polymer gets easily adsorbed on an Au nanosurface via $\mathrm{OH}$ and $>\mathrm{C}=\mathrm{O}$ sites of a $\mathrm{PVA}$ polymer of thin surface layer in a core-shell structure. Small $\xi$ value in our samples (i.e., less than $-30.0 \mathrm{mV}$ ) suggests that colloidal stability is due to both electrostatic and steric stabilization mechanisms. In an experiment, Rahme et al. [39] found that the $\xi$ value in a nanofluid also depends on the host structure. A $\xi$ value had

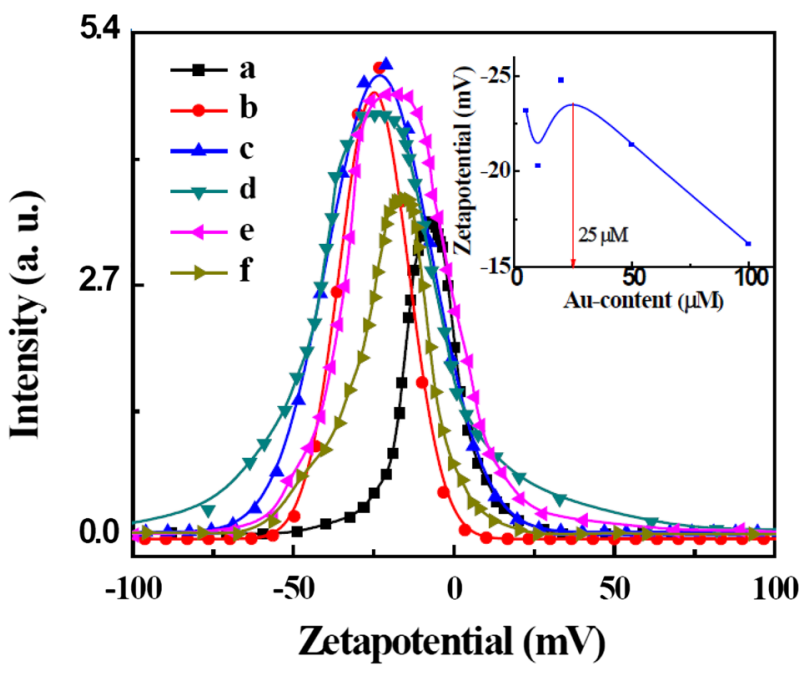

Fig. 9 Distribution of $\xi$ values in Au-PVA NFs consisting of $a 0, b 5$, $c 10, d 20, e$ 50, and $f 100 \mu \mathrm{M}$ NG along with $30.0 \mathrm{~g} / \mathrm{L}$ PVA in water. Inset shows variations in $\xi$ value with $\mathrm{Au}$-content been decreased from $(-) 35 \mathrm{mV}$ to $(-) 1 \mathrm{mV}$ in a goldreinforced PEG nanofluid in a nonlinear path when PEG molecular weight was increased from 2100 to $51,400 \mathrm{~g} \mathrm{~mol}^{-1}$. They remarked that such decrease in the $\xi$ value with an increased molecular weight of a liquid carrier is not surprising as zeta potential is measured at the surface plane of the hydrodynamic sphere of diameter and the surface plane of the hydrodynamic sphere is far from the surface of flocculates in a stable nanofluid. PEG-capped $\mathrm{Au}$ NPs exhibit an average $\xi$ value as $(-) 13.9 \mathrm{mV}$ for the highest loading of polymer as a result of steric stabilization compared to a large $\xi$ value of $(-) 39.5 \mathrm{mV}$ shown in citrate-capped $\mathrm{Au}$ NPs [41].

\section{Surface encapsulation study by microstructure and absorption spectra in Au-PVA NF}

Now let us examine HRTEM images (Fig. 10) from a representative PVA-modified NG in the nanofluid, which are processed with $5.0 \mu \mathrm{M}$ Au with $30.0 \mathrm{~g} / \mathrm{L} \mathrm{PVA}$ in water. As seen from the image, the NGs are coated with PVA in such a way that they appear mostly thin plates of various architectures such as hexagons, pentagons, triangles, and heptagons of 20-25 nm diameters. A typical hexagonal plate of PVA-encapsulated Au-NP demonstrates that a distinct surface layer of PVA adheres firmly to the darkish region of the NG. Rahme et al. [39] have reported coreshell structures in triblock copolymer-capped Au-NPs. In an another experiment, a similar kind of organic-inorganic hybrid nanostructure is reported in Ag-NPs coated with polystyrene/methyl methacrylate [42]. Gold NPs were coated with polyelectrolyte using Layer-by-Layer deposition method in order to develop nanostructure of various architecture so that the resulting core-shell NPs are so well
Fig. 10 Typical TEM images (a) taken from 5.0 $\mu \mathrm{M}$ NG with $30 \mathrm{~g} / \mathrm{L}$ PVA in water. Lattice fringes in the inset reveal crystalline nature of PVAencapsulated NG. Rings of an electron diffraction pattern (b) of NG correspond to (111), (200), (220), and (311) planes of fcc Au atom

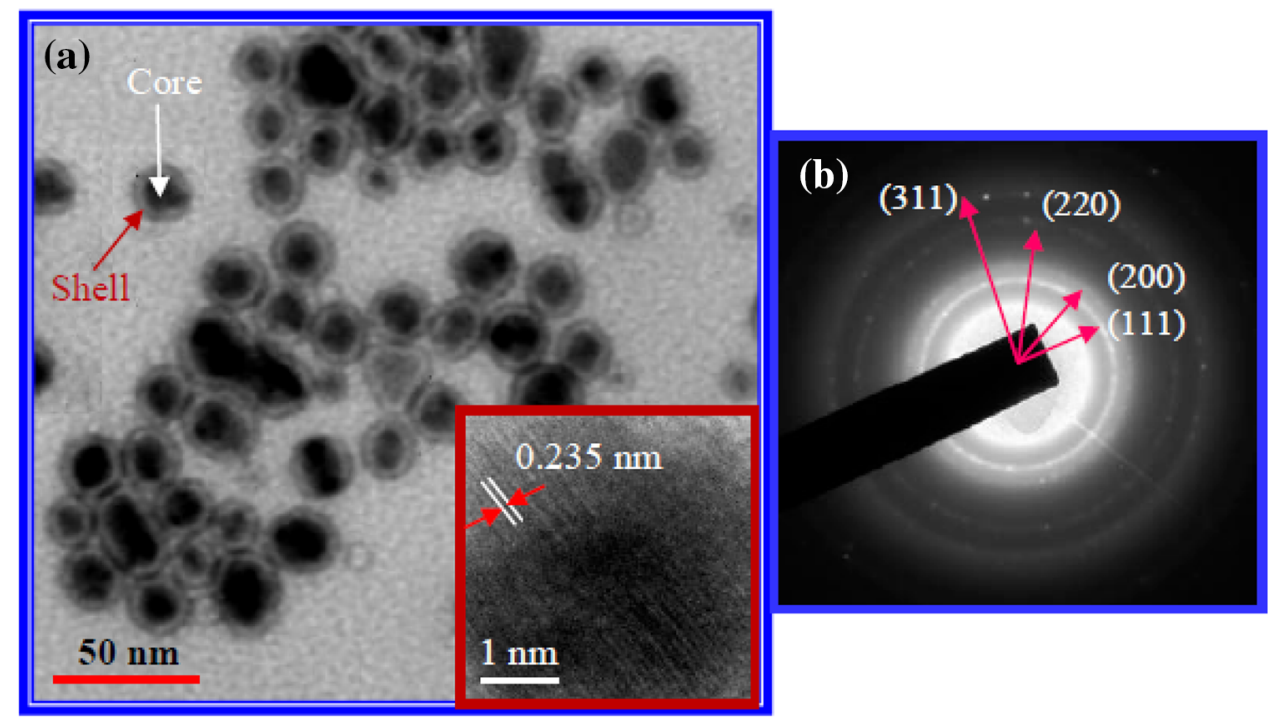




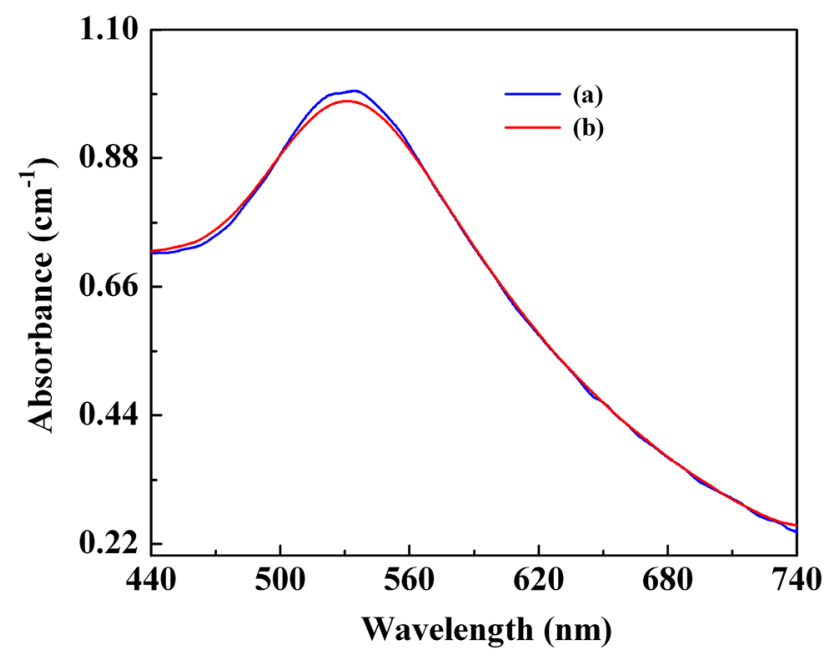

Fig. 11 Absorption spectra of $10 \mu \mathrm{M}$ Au-PVA nanofluid $a$ before and $b$ after addition of $10 \% \mathrm{NaCl}$ electrolyte in water

stabilized in a nanofluid $[17,18]$. Our groups have already reported such core-shell structures in Au NPs capped in PVP in both aqueous and non-aqueous carriers [20-24]. A high-resolution TEM image of PVA-encapsulated NG displays lattice fringes and confirms that crystalline structure of NG with inter planar spacing $0.235 \mathrm{~nm}$ corresponds to (111) plane $[16,19]$. The polycrystalline nature of Au-NP was confirmed by diffraction rings (b) obtained from bulk $\mathrm{Au}-\mathrm{NP}$. Those electron diffraction rings could be assigned to (111), (200), (220), and (311) planes of face-centered cubic $\mathrm{Au}[16,19-24,43]$. To confirm encapsulation of NG by PVA polymer, an aqueous solution of $\mathrm{NaCl}(10 \%)$ electrolyte was added to Au-PVA nanofluid and mixed in a magnetic stirrer for $10 \mathrm{~min}$. No precipitates were detected upon mechanical agitation in the presence of this strong electrolyte. To further confirm, we studied the absorption spectra (Fig. 11) of Au-PVA nanofluid before and after the addition of the electrolyte. Figure 11 shows that the $\lambda_{\max }$ of the SPR band remains unaffected in the presence of the electrolyte, revealing efficient encaging of $\mathrm{NG}$ by the polymer but the intensity has decreased by $\sim 8 \%$ owing to adsorption of the electrolyte on the surface of the NPs [44].

\section{Conclusion}

Gold-PVA NFs were developed in an aqueous medium via a chemical reduction route at as low temperature as $40{ }^{\circ} \mathrm{C}$ using gold nitrate as precursor salt. It has been found that $\lambda_{\max }$ of the SPR band increases as PVA-capped NG cluster size increases with Au-content. FTIR spectra suggest existence of an interfacial interaction between $\mathrm{C}=\mathrm{O}$ group of oxidized PVA and surface of NG. A scheme was proposed to support amplification in the band intensity of $-\mathrm{C}=\mathrm{O}$ group in the PVA polymer in presence of NG. Electrokinetic study of the Au-PVA NFs further reveals formation of assemblies between $\mathrm{Au}$ and PVA via interaction between $\mathrm{C}=\mathrm{O}$ group of PVA and NG. From the study, it is found that a strong correlation exists between optical and electrokinetic parameters in the Au-PVA NFs. HRTEM images validate the optical, rheological, and electrokinetic study on the NFs. Development of core-shell nanostructure of Au-PVA NFs in an aqueous medium via a green route could find applications in the field of biosensors.

Acknowledgments This work has been supported by the Silicon Institute of Technology, Silicon Hills, Bhubaneswar, India.

Open Access This article is distributed under the terms of the Creative Commons Attribution 4.0 International License (http:// creativecommons.org/licenses/by/4.0/), which permits unrestricted use, distribution, and reproduction in any medium, provided you give appropriate credit to the original author(s) and the source, provide a link to the Creative Commons license, and indicate if changes were made.

\section{References}

1. Choi, S.U.S.: Enhancing thermal conductivity of fluids with nanoparticles. In: Siginer, D.A., Wang, H.P. (eds.) Developments and applications of non-Newtonian flows, FED-231/MD, pp. 99-105. ASME, New York (1995)

2. Das, S.K., Choi, S.U.S., Yu, W., Pradeep, T.: Nanofluids: Science and technology. Wiley, London (2007)

3. Wang, L.Q., Quintard, M.: Nanofluids of the future. Advances in transport phenomena, pp. 179-243. Heidelberg, Berlin (2009)

4. Wang, L.Q., Fan, J.: Nanofluids research: key issues. Nanoscale Res. Lett. 5, 1241-1252 (2010)

5. Ghadimi, A., Saidur, R., Metselaar, H.S.C.: A review of nanofluid stability properties and characterization in stationary conditions. Int J Heat Mass Transf. 54, 4051-4068 (2011)

6. Yu, W., Xie, H.: A review on nanofluids: preparation, stability mechanisms, and application. J Nanomater 2012, 435873 (2012)

7. Philip, J., Shima, P.D.: Thermal properties of nanofluids. Adv. Colloid Interface Sci. 183, 30-45 (2012)

8. Faraday, M.: Experimental relations of gold (and other metals). Philos Trans R. Soc London 147, 145-181 (1857)

9. Porel, S., Singh, S., Radhakrishnan, T.P.: Polygonal gold nanoplates in a polymer matrix. Chem. Commun. 18, 2387-2389 (2005)

10. Abyaneh, M.K., Parmanik, D., Varma, S., Gosavi, S.W., Kulkarni, S.K.: Formation of gold nanoparticles in polymethylmethacrylate by UV irradiation. J. Phys. D Appl. Phys. 40, 3771-3779 (2007)

11. Shi, H., Xu, N., Zhao, D., Xu, B.Q.: Immobilized PVA-stabilized gold nanoparticles on silica show an unusual selectivity in the hydrogenation of cinnamaldehyde. Catal. Commun. 9, 1949-1954 (2008)

12. Mahendia, S., Tomar, A.K., Chahal, R.P., Goyal, P., Kumar, S.: Optical and structural properties of poly (vinyl alcohol) films embedded with citrate-stabilized gold nanoparticles. J. Phys. D Appl. Phys. 44, 205105 (2011)

13. Alexandridis, P.: Gold nanoparticle synthesis, morphology control, and stabilization facilitated by functional polymers. Chem. Engg. Technol. 34, 15-28 (2011) 
14. Shalklvicht, N., Escher, W., Burgi, T., Michel, B., Si-Ahmed, L., Poulikakos, D.: On the thermal conductivity of gold nanoparticle colloids. Langmuir 26, 663-670 (2010)

15. Zhang, H., Wu, Q., Lin, J., Chen, J., Xu, Z.: Thermal conductivity of polyethylene glycol nanofluids containing carbon coated metal nanoparticles. J. Appl. Phys. 108, 124304-124309 (2010)

16. Tripathy, P., Mishra, A., Ram, S., Fecht, H.J., Bansmann, J., Behm, R.J.: X-ray photoelectron spectrum in surface interfacing of gold nanoparticles with polymer molecules in a hybrid nanocomposite structure. Nanotechnology 20, 075701 (2009)

17. Schneider, G., Decher, G.: From functional core/shell nanoparticles prepared via layer-by-layer deposition to empty nanospheres. Nano Lett. 4, 1833-1839 (2004)

18. Pereira, S.O., Barros-Timmons, A., Trindade, T.: Biofunctionalisation of colloidal gold nanoparticles via polyelectrolytes assemblies. Colloid Polym. Sci. 292, 33-50 (2014)

19. Ram, S., Fecht, H.-J.: Modulating up-energy transfer and violetblue light emission in gold nanoparticles with surface adsorption of poly(vinyl pyrrolidone) molecules. J. Phys. Chem. C. 115, 7817-7828 (2011)

20. Behera, M., Ram, S.: Synthesis of gold nanoparticles in presence of poly(vinyl pyrrolidone) from gold hydroxide precursor salt. Adv. Mat. Res. 585, 115-119 (2012)

21. Behera, M., Ram, S.: Synthesis and characterization of core-shell gold nanoparticles with poly(vinyl pyrrolidone) from a new precursor salt. Appl. Nanosci. 3, 83-87 (2013)

22. Behera, M., Ram, S.: Intense quenching of fluorescence intensity of poly(vinyl pyrrolidone) molecules in presence of gold nanoparticles. Appl. Nanosci. 3, 543-548 (2013)

23. Behera, M., Ram, S.: Spectroscopic based study on the interaction between gold nanoparticle and poly(vinyl pyrrolidone) molecules in a non-hydrocolloid. Int. Nano Lett. 3, 1-7 (2013)

24. Behera, M., Ram, S.: Inquiring the mechanism of formation, encapsulation, and stabilization of gold nanoparticles by Poly(vinyl pyrrolidone) molecules in 1-butanol. Appl. Nanosci. 4, 247-254 (2014)

25. Wang, W., Zhang, S., Srisombat, L.O., Lee, R.T., Advincula, R.C.: Gold-nanoparticle- and gold-nanoshell-induced polymorphism in poly(vinylidene fluoride). Macromol. Mater. Eng. 296, 178-184 (2011)

26. Mandal, D., Henkel, K., Schmeißer, D.: The Electroactive $\beta$ phase formation in poly(vinylidene fluoride) by gold nanoparticles doping. Mater. Lett. 73, 123-125 (2012)

27. Cosgrove, T.: Colloid science: principle, methods, and applications. Wiley, London (2010)

28. Hunter, R.J.: Zeta potential in colloid science: principles and applications. Academic Press, UK (1988)

29. Ram, S., Mandal, T.K.: Photoluminescence in small isotactic, atactic and syndiotactic PVA polymer molecules in water. Chem. Phys. 303, 121-128 (2004)
30. Kreibig, U., Vollmer, M.: Optical properties of metal clusters. Springer, Berlin (1996)

31. Perez-Juste, J., Pastoriza-Santos, I., Liz-Marźan, L.M., Mulvaney, P.: Gold nanorods: synthesis, characterization and applications. Coord. Chem. Rev. 249, 1870-1901 (2005)

32. Tawansi, A., El-Khodary, A., Abdelnaby, M.M.: A study of the physical properties of $\mathrm{FeCl}_{3}$ filled PVA. Curr. App. Phys. 5, 572-578 (2005)

33. Sun, C., Qu, R., Ji, C., Meng, Y., Wang, C., Sun, Y., Qi, L.: Preparation and property of polyvinyl alcohol-based film embedded with gold nanoparticles. J. Nanopart. Res. 11, 1005-1010 (2009)

34. Bhatia, P., Yadav, P., Gupta, B.D.: Surface plasmon resonance based fiber optic hydrogen peroxide sensor using polymer embedded nanoparticles. Sens. Actuat. B Chem. 182, 330-335 (2013)

35. Grace, A.N., Pandian, K.: One pot synthesis of polymer protected gold nanoparticles and nanoprisms in glycerol. Colloids Surf. A 290, 138-142 (2006)

36. Mishra, A., Ram, S., Ghosh, G.: Dynamic light scattering and optical absorption in biological nanofluids of gold nanoparticles in poly(vinyl pyrrolidone) molecules. J. Phys. Chem. C 113, 6976-6982 (2009)

37. Phule, A.D., Ram, S., Tyagi, A.K.: Anchoring silver with poly(vinylidene fluoride) molecules in model flocculates and its effects on rheology in stable nanofluids. J. Nanofluids 2, 249-260 (2013)

38. Muller, S., Llewellin, E.W., Mader, H.M.: Rheology of suspensions of solid particles. Proc R. Soc. A 466, 1201-1228 (2010)

39. Rahme, K., Oberdisse, J., Schweins, R., Gaillard, C., Marty, J.D., Mingotaud, C., Gauffre, F.: Pluronics-stabilized gold nanoparticles: investigation of the structure and the polymer-particle hybrid. Chem. Phys. Chem. 9, 2230-2236 (2008)

40. Behera, M., Ram, S.: Solubilization and stabilization of fullerene $\mathrm{C}_{60}$ in presence of poly (vinyl pyrrolidone) molecules in water. J. Incl. Phenom. Macrocycl. Chem. 72, 233-239 (2012)

41. Manson, J., Kumar, D., Meenan, B.J.: Polyethylene glycol functionalized gold nanoparticles: the influence of capping density on stability in various media. Gold Bull. 44, 99-105 (2011)

42. Quaroni, L., Chumanov, G.: Preparation of polymer-coated functionalized silver nanoparticles. J. Am. Chem. Soc. 121, 10642-10643 (1999)

43. Kemal, L., Jiang, X.C., Wong, K., Yu, A.B.: Experiment and theoretical study of poly(vinyl pyrrolidone)-controlled gold nanoparticles. J. Phys. Chem. C 112, 15656-15664 (2008)

44. Bashir, O., Hussain, S., Khan, Z., AL-Thabaiti, S.A.: Encapsulation of silver nanocomposites and effects of stabilizers. Carbohydr. Polym. 107, 167-173 (2014) 